ANALYSIS \& PDE Volume 6 No. 5 2013 SW YVES COLIN DE VERDIERE MAGNETIC INTERPRETATION OF THE NODAL DEFECT ON GRAPHS 


\title{
MAGNETIC INTERPRETATION OF THE NODAL DEFECT ON GRAPHS
}

\author{
YVES COLIN DE VERDIÈRE
}

We present a natural proof of a recent and surprising result of Gregory Berkolaiko interpreting the Courant nodal defect as a Morse index. This proof is inspired by a nice paper of Miroslav Fiedler published in 1975.

\section{Introduction}

The "nodal defect" of an eigenfunction of a Schrödinger operator is closely related to the difference between the upper bound on the number of nodal domains given by Courant's theorem and the number of nodal domains. Berkolaiko [2013] has proved a nice formula for the nodal defect of an eigenfunction of a Schrödinger operator on a finite graph in terms of the Morse index of the corresponding eigenvalue as a function of a magnetic deformation of the operator. His proof remains mysterious and rather indirect. In order to get a better understanding in view of possible generalizations, it is desirable to have a more direct approach. This is what we do here, with a proof inspired by [Fiedler 1975].

After reviewing our notations, we state the main result, as well as a reinterpretation in terms of Hessians of a determinant, and give an informal description of the proof in Section 3. The proof itself is implemented in Sections 4 and 5 with an alternative view provided in Appendix A. The continuous Schrödinger operator on a circle was considered in the preprint version of this paper [Colin de Verdière 2012]. The case of quantum graphs, i.e., graphs as 1-dimensional simplicial complexes, is worked out in [Berkolaiko and Weyand 2012].

\section{Notation}

Let $G=(X, E)$ be a finite connected graph, where $X$ is the set of vertices and $E$ the set of unoriented edges. We denote by $\{x, y\}$ the edge linking the vertices $x$ and $y$. We denote by $\vec{E}$ the set of oriented edges and by $[x, y]$ the edge from $x$ to $y$; the set $\vec{E}$ is a 2-fold cover of $E$. A 1-form $\alpha$ on $G$ is a map $\vec{E} \rightarrow \mathbb{R}$ such that $\alpha([y, x])=-\alpha([x, y])$ for all $\{x, y\} \in E$. We denote by $\Omega^{1}(G)$ the vector space of dimension \#E of 1-forms on $G$. The operator $d: \mathbb{R}^{X} \rightarrow \Omega^{1}(G)$ is defined by $d f([x, y])=f(y)-f(x)$. If $Q$ is a nondegenerate, not necessarily positive, quadratic form on $\Omega^{1}(G)$, we denote by $d^{\star}$ the adjoint of $d$, where $\mathbb{R}^{X}$ carries the canonical Euclidean structure and $\Omega^{1}(G)$ is equipped with the symmetric inner product $\hat{Q}$ associated to $Q$. We have $\operatorname{dim} \operatorname{ker} d^{\star}=\beta$, where $\beta=1+\# E-\# X$ is the dimension

MSC2010: 05C50, 35P05.

Keywords: operator on graphs, Schrödinger operators with magnetic fields, Morse index, Courant nodal theorem, Hill's operator. 
of the space of cycles of $G$. We will show later that, in our context, we have the Hodge decomposition $\Omega^{1}(G)=d \mathbb{R}^{X} \oplus \operatorname{ker} d^{\star}$, where both spaces are $\hat{Q}$-orthogonal.

Following [Colin de Verdière 1998], we denote by $O_{G}$ the set of $X \times X$ real symmetric matrices $H$ which satisfy $h_{x, y}<0$ if $\{x, y\} \in E$ and $h_{x, y}=0$ if $\{x, y\} \notin E$ and $x \neq y$. Note that the diagonal entries of $H$ are arbitrary. An element $H$ of $O_{G}$ is called a Schrödinger operator on the graph $G$. It will be useful to write the quadratic form associated to $H$ as

$$
q_{1}(f)=-\sum_{\{x, y\} \in E} h_{x, y}(f(x)-f(y))^{2}+\sum_{x \in X} V_{x} f(x)^{2},
$$

with $V_{x}=h_{x, x}+\sum_{y \sim x} h_{x, y}$. A magnetic field on $G$ is a map $B: \vec{E} \rightarrow U(1)$ defined by $B([x, y])=e^{i \alpha_{x, y}}$, where $[x, y] \mapsto \alpha_{x, y}$ is a 1-form on $G$. We denote by $\mathscr{B}_{G}=e^{i \Omega^{1}(G)}$ the manifold of magnetic fields on $G$. The magnetic Schrödinger operator $H_{B}$ associated to $H \in \mathscr{O}_{G}$ and $B=e^{i \alpha}$ is defined by the quadratic form

$$
q_{B}(f)=-\frac{1}{2} \sum_{[x, y] \in \vec{E}} h_{x, y}\left|f(x)-e^{i \alpha_{x, y}} f(y)\right|^{2}+\sum_{x \in X} V_{x}|f(x)|^{2}
$$

associated to a Hermitian form on $\mathbb{C}^{X}$. More explicitly, if $f \in \mathbb{C}^{X}$,

$$
H f(x)=h_{x, x} f(x)+\sum_{y \sim x} h_{x, y} e^{i \alpha_{x, y}} f(y) .
$$

We fix $H$ and we denote by

$$
\lambda_{1}(B) \leq \lambda_{2}(B) \leq \cdots \leq \lambda_{n}(B) \leq \cdots \leq \lambda_{\# X}(B)
$$

the eigenvalues of $H_{B}$. It will be important to notice that $\lambda_{n}(\bar{B})=\lambda_{n}(B)$. Moreover, we have a gauge invariance: the operators $H_{B}$ and $H_{B^{\prime}}$ with $\alpha^{\prime}=\alpha+d f$ for some $f \in \mathbb{R}^{X}$ are unitarily equivalent. Hence they have the same eigenvalues. This implies that, if $\Omega^{1}(G)=d \mathbb{R}^{X} \oplus \operatorname{ker} d^{\star}$ (this is not always the case because $Q$ is not positive), it is enough to consider 1-forms in the subspace $\operatorname{ker} d^{\star}$ of $\Omega^{1}(G)$ when studying the map $\Lambda_{n}: B \rightarrow \lambda_{n}(B)$. This holds in particular for investigations concerning the Hessian and the Morse index.

\section{Statement of Berkolaiko's magnetic theorem}

Before stating the main result, we recall:

Definition 1. The Morse index $j(q) \in \mathbb{N} \cup\{+\infty\}$ of a quadratic form $q$ on a real vector space $E$ is defined by $j(q)=\sup _{F} \operatorname{dim} F$, where $F$ is a subspace of $E$ such that $q_{\mid F \backslash 0}$ is less than 0 . The nullity of $q$ is the dimension of the kernel of $q$.

The Morse index of a smooth real-valued function $f$ defined on a smooth manifold $M$ at a critical point $x_{0} \in M$ (i.e., a point satisfying $d f\left(x_{0}\right)=0$ ) is the Morse index of the Hessian of $f$, which is a canonically defined quadratic form on the tangent space $T_{x_{0}} M$. The critical point $x_{0}$ is called nondegenerate if the previous Hessian is nondegenerate. The nullity of the critical point $x_{0}$ of $f$ is the nullity of the Hessian of $f$ at the point $x_{0}$. 
The aim of this note is to prove the following nice results due to Berkolaiko [2008; 2013]:

Theorem 1. Let $G=(X, E)$ be a finite connected graph and $\beta$ the dimension of the space of cycles of $G$. We suppose that the $n$-th eigenvalue $\lambda_{n}$ of $H \in \mathrm{O}_{G}$ is simple. We assume moreover that an associated nonzero eigenfunction $\phi_{n}$ satisfies $\phi_{n}(x) \neq 0$ for all $x \in X$. Then, the number $v$ of edges along which $\phi_{n}$ changes sign satisfies $n-1 \leq v \leq n-1+\beta$.

Moreover $\Lambda_{n}: B \rightarrow \lambda_{n}(B)$ is smooth at $B \equiv 1$ which is a critical point of $\Lambda_{n}$ and the nodal defect, $\delta_{n}=v-(n-1)$, is the Morse index of $\Lambda_{n}$ at that point. If $M$ is the manifold of dimension $\beta$ of magnetic fields on $G$ modulo the gauge transforms, the function $[B] \rightarrow \Lambda_{n}(B)$ has $[B=1]$ as a nondegenerate critical point.

Remark 1. The previous results can be extended by replacing the critical point $B \equiv 1$ by $B_{x, y}= \pm 1$ for all edges $\{x, y\} \in E$. The number $v$ is then the number of edges $\{x, y\} \in E$ satisfying $B_{x, y} \phi_{n}(x) \phi_{n}(y)<0$ where $\phi_{n}$ is the corresponding eigenfunction.

Remark 2. The assumptions on $H$ are satisfied for $H$ in an open dense subset of $O_{G}$.

The upper bound of $v$ in the first part of Theorem 1 is related to the Courant nodal theorem (see [Courant and Hilbert 1953, Section VI.6]) as follows: a nodal domain on a graph for the eigenfunction $\phi_{n}$ is a connected component of the subgraph $G^{\prime}$ of $G$ obtained by removing the edges along which $\phi_{n}$ changes sign. Denoting by $\mu$ the number of nodal domains of $\phi_{n}$, the Courant theorem for graphs (see [Colin de Verdière 1998, Theorem 2.4]) asserts that $\mu \leq n$; using the Euler formula for the graph $G^{\prime}$ and because $\mu=b_{0}\left(G^{\prime}\right)$, the number of connected components of the graph $G^{\prime}$, we get also a lower bound (see [Berkolaiko 2008]):

Corollary 1. Under the assumptions of Theorem 1, we have $n-\beta \leq \mu \leq n$.

Example 3.1 (bipartite graphs). Let $G=(V, E)$ be a bipartite graph: $V=Y \cup Z$ and all edges have one vertex in $Y$ and the other in $Z$. Let $U$ be the involution on $\mathbb{R}^{V}$ given by $U f(x)=-f(x)$ if $x \in Y$ and $U f(x)=f(x)$ if $x \in Z$ and let $B$ be a magnetic field. Then $U H_{B} U=-H_{B}^{\prime}$ with $H^{\prime} \in O_{G}$, so that $\lambda_{|V|}\left(H_{B}\right)=-\lambda_{1}\left(H_{B}^{\prime}\right)$. And hence it follows from the diamagnetic inequality that $B \rightarrow \lambda_{|V|}\left(H_{B}\right)$ has a maximum at $B \equiv 1$. And hence the Morse index of the Hessian of $B \rightarrow \lambda_{|V|}\left(H_{B}\right)$ at $B \equiv 1$ is the dimension of the manifold of magnetic fields, namely $\beta$. On the other hand the first eigenfunction $\phi_{1}$ of $H^{\prime}$ is everywhere greater than 0 and the number of sign changes of $U \phi_{1}$ is $|E|$. So Berkolaiko's formula for $\lambda_{|V|}$ gives $(|V|-1)+\beta=|E|$. This is the Euler formula.

Theorem 1 can be reinterpreted as follows:

Theorem 2. Under the assumptions as in Theorem 1, consider the functional $D_{n}: B \mapsto \operatorname{det}\left(H_{B}-\lambda_{n}(1)\right)$. Then $B \equiv 1$ is a nondegenerate critical point of $D_{n}$ whose Morse index is $\delta_{n}$ if $n$ is odd and $\beta-\delta_{n}$ if $n$ is even.

Proof. Under the assumptions of the theorem we have

$$
\operatorname{det}\left(H_{B}-\lambda_{n}(1)\right)=\left(\lambda_{n}(B)-\lambda_{n}(1)\right) \operatorname{det}^{\prime}\left(H_{B}-\lambda_{n}(1)\right)
$$


where $\operatorname{det}^{\prime}\left(H_{B}\right)=F(B)$ is the product of the eigenvalues $\lambda_{j}-\lambda_{n}(1)$ for $j \neq n$. The following lemma is easy to check by direct computations of the second derivatives:

Lemma 1. Let $F=f G$ where $F, f, G$ are smooth real valued functions defined near a point $x_{0}$ on a smooth manifold. Let us assume that $f\left(x_{0}\right)=0$ and $f^{\prime}\left(x_{0}\right)=0$; then the Hessian of $F$ at the point $x_{0}$ is $G\left(x_{0}\right)$ times the Hessian of $f$ at $x_{0}$.

From the lemma, we get that the Hessian of $D_{n}$ at $B \equiv 1$ is $F(1)$ times the Hessian of $\Lambda_{n}$. We have $(-1)^{n-1} F(1)>0$. The conclusion follows.

There is a formula for the characteristic polynomial of a magnetic Laplacian on graphs due to Robin Forman [1993] and reproved by Richard Kenyon [2012] and Yurii Burman [2012]. Using the gauge change $f \rightarrow f \phi_{n}$ as in [Colin de Verdière 1998] gives a Laplace type operator whose entries can be of any sign. Forman's formula extends to that case and it would be nice to relate Berkolaiko's formula to Forman's formula.

Important warning: Without loss of generality, we can and will assume in the rest of this note that $\lambda_{n}=\Lambda_{n}(1)=0$. This implies that the Morse index of $q_{1}$ is $n-1$.

In the course of the proof we will use a special choice of gauge in which we can compute the Hessian explicitly. More precisely, according to the classical perturbation formulae,

$$
\ddot{\lambda}=(\phi, \ddot{H} \phi)+2(\dot{H} \phi, \dot{\phi}),
$$

where we assumed that $\lambda$ is at a critical point: $\dot{\lambda}=0$. The first term is easy to calculate explicitly; for perturbation in the direction of the 1 -form $\omega$ it is

$$
Q(\omega)=\frac{1}{2} \sum_{\vec{E}} a_{x, y} \omega([x, y])^{2} \quad \text { with } a_{x, y}=-h_{x, y} \phi_{n}(x) \phi_{n}(y)=a_{y, x} .
$$

Considered as a quadratic form in $\omega, Q$ is already in the diagonal form. Its index is clearly the number of negative values among $\left\{-h_{x, y} \phi_{n}(x) \phi_{n}(y)\right\}$, or, in other words, the number $v$ of edges where $\phi_{n}$ changes sign!

We will present an explicit choice of gauge in which the second term vanishes. The condition for this is $\dot{H} \phi=0$ which, after explicit calculation, can be interpreted as $\omega \in \operatorname{ker} d^{\star}$, where $d^{\star}$ is the conjugate of $d$ with respect to the inner product induced by (2).

Finally, we observe that the index of $Q(\omega)$ has been computed to be $v$ in the whole of $\Omega^{1}(G)$, whereas we should be restricting ourselves to our chosen gauge, $\omega \in \operatorname{ker} d^{\star}$. We will show that this restriction reduces the index precisely by $n-1$. Indeed, the splitting $\Omega^{1}(G)=d \mathbb{R}^{X} \oplus \operatorname{ker} d^{\star}$ is orthogonal with respect to the form $Q$; therefore

$$
\operatorname{ind}(Q)=\operatorname{ind}\left(\left.Q\right|_{d \mathbb{R}^{X}}\right)+\operatorname{ind}\left(\left.Q\right|_{\operatorname{ker} d^{\star}}\right) .
$$

We establish that ind $\left(\left.Q\right|_{d \mathbb{R}^{X}}\right)=n-1$ by relating the form $Q$ on $d \mathbb{R}^{X}$ to the quadratic form $q_{1}$ around the point $\phi_{n}$. 


\section{The quadratic form $Q$}

Lemma 2. The set of forms $f \rightarrow(f(x)-f(y))^{2}$ where $\{x, y\} \in \mathscr{P}_{2}(X)$, the set of subsets with two elements of $X$, and $f \rightarrow f(x)^{2}$ with $x \in X$ is a basis of the set of quadratic forms on $\mathbb{R}^{X}$.

Definition 2. A quadratic form $q$ on $\mathbb{R}^{X}$ is said of Laplace type if for all $f \in \mathbb{R}^{X}, \hat{q}(1, f) \equiv 0$ where $\hat{q}$ is the symmetric bilinear form associated to $q$.

Lemma 3. The set of forms $f \rightarrow(f(x)-f(y))^{2},\{x, y\} \in \mathscr{P}_{2}(X)$ is a basis of the space of quadratic forms of Laplace type.

The form $\tilde{q}_{1}: f \rightarrow q_{1}\left(\phi_{n} f\right)$, where $\phi_{n} f$ is the pointwise product of $\phi_{n}$ and $f$, is of Laplace type because

$$
\widehat{\tilde{q}_{1}}(1, g)=\left\langle H \phi_{n} \mid \phi_{n} g\right\rangle=\left\langle 0 \mid \phi_{n} g\right\rangle .
$$

Hence $\widehat{\tilde{q}_{1}}(1, g)=0$.

Moreover, $\tilde{q}_{1}(f)=Q(d f)$. Indeed, because of Lemma 3, it is enough to compare the coefficients of the basis forms $f \rightarrow(f(x)-f(y))^{2}$. The form $f \rightarrow Q(d f)$ is already expanded in this basis. To find the coefficient for the form $f \rightarrow \tilde{q}_{1}(f)$, we observe that (because we know it is of Laplace type) the coefficient in question is minus the coefficient in front of the term $f(x) f(y)$, divided by two. This evaluates to $a_{x, y}$ (see (2)).

In fact, we will need to use $\hat{Q}(d f, d g)=\left\langle H\left(\phi_{n} f\right) \mid \phi_{n} g\right\rangle$.

Lemma 4. The Morse index of $Q_{\mid d \mathbb{R}^{X}}$ is equal to $n-1$.

It is a general fact that the Morse index of the quadratic form $f \rightarrow Q(A f)$ is the same as the Morse index of the restriction of $Q$ to the image of $A$. Hence, the Morse index of $Q_{\mid d \mathbb{R}^{X}}$ is the Morse index of $\tilde{q}_{1}$ on $\mathbb{R}^{X}$. Because $f \rightarrow \phi_{n} f$ is a linear isomorphism, this index is equal to the index of $q_{1}$ by the Sylvester theorem. Since $\lambda_{n}=0$, the index of $q_{1}$ is $n-1$ by elementary spectral theory.

Lemma 5. Let us denote by $d^{\star}$ the adjoint of $d$ where $\mathbb{R}^{X}$ is equipped with the canonical Euclidean structure and $\Omega^{1}(G)$ with the inner product associated to $Q$. The space $\Omega^{1}(G)$ splits as

$$
\Omega^{1}(G)=d \mathbb{R}^{X} \oplus \operatorname{ker} d^{\star}
$$

(Hodge type splitting), and this decomposition is Q-orthogonal.

More explicitly $d^{\star}$ is given by

$$
d^{\star} \omega(x)=\sum_{y \sim x} a_{x, y} \omega([y, x]) .
$$

If $\omega=d f$ satisfies $d^{\star} \omega=0$, we have $d^{\star} d f=0$. Hence $\hat{Q}(d f, d g)=0$ for all $g$ and $\left.\left.\left\langle H\left(\phi_{n} f\right)\right| \phi_{n} g\right)\right\rangle=0$. Because $\lambda_{n}$ is of multiplicity 1 , this implies that $f$ is constant and hence $d f=0$. So $d \mathbb{R}^{X} \cap \operatorname{ker} d^{\star}=\{0\}$ and the conclusions follow.

At this point, we know that the nodal defect is the Morse index of the restriction of $Q$ to the space $\operatorname{ker} d^{\star}$ of dimension $\beta$. The first part of Theorem 1 follows. 


\section{The magnetic Hessian}

We need one more fact to complete the proof: to identify the Hessian of $\Lambda_{n}$ on $e^{i \operatorname{ker} d^{\star}}$ at $B \equiv 1$ with the restriction of $Q$ to $\operatorname{ker} d^{\star}$.

Let us denote by $S \subset \mathbb{C}^{X}$ the set of unit vectors $f$ normalized so that $f\left(x_{0}\right)$ is real and $f\left(x_{0}\right)>0$ where $x_{0}$ is chosen in $X$.

Lemma 6. The point $B \equiv 1$ is a critical point of $\Lambda_{n}$. If $\phi_{n}(B) \in S$ is the eigenfunction of $H_{B}$ corresponding to the eigenvalue $\lambda_{n}(B)$, the differential of $B \rightarrow \phi_{n}(B)$ vanishes at $B \equiv 1$ on $\operatorname{ker} d^{\star}$.

The first property comes from the fact that $\Lambda_{n}(\bar{B})=\Lambda_{n}(B)$. We can compute, for any variation $e^{i t \alpha}$, $t$ close to 0 , of $B \equiv 1$, that $\dot{H}_{B} \phi_{n}+H \dot{\phi}_{n}=0$. The condition $d^{\star} \alpha=0$ can be written as

$$
\sum_{y \sim x} h_{x, y} \phi_{n}(y) \alpha_{x, y}=0 \quad \text { for all } x \in X
$$

From (1), this is equivalent to $\dot{H}_{B} \phi_{n}=0$. Hence $H\left(\dot{\phi}_{n}\right)=0$ and $\dot{\phi}_{n}=c \phi_{n}$ since $\lambda_{n}$ is simple. From the normalization $\left\|\phi_{n}(B)\right\|=1$, we get $c \in i \mathbb{R}$ and, since $\dot{\phi}_{n}\left(x_{0}\right) \in \mathbb{R}$, the number $c$ is real. We deduce that $\dot{\phi}_{n}=0$.

Lemma 7. The function $F: S \times e^{i \operatorname{ker} d^{\star}} \rightarrow \mathbb{R}$ defined by $F\left(f, e^{i \alpha}\right)=\left\langle H_{e^{i \alpha}} f \mid f\right\rangle$ admits $\left(\phi_{n}, 0\right)$ as a critical point and the Hessian of $\left(\Lambda_{n}\right)_{\mid e^{i \mathrm{ker} d^{\star}}}$ at the point $B \equiv 1$ is the form $Q$.

The differential of $F$ with respect to $f$ vanishes because $f$ is an eigenfunction of $H$. The differential with respect to ker $d^{\star}$ vanishes, because $F\left(f, e^{i \alpha}\right)=F\left(f, e^{-i \alpha}\right)$. The Hessian of $F$ at $\left(\phi_{n}, 0\right)$ is well defined. Because the differential at $B=1$ of $B \rightarrow \phi_{n}(B)$ vanishes on $e^{i \operatorname{ker} d^{\star}}$, the Hessians of $\Lambda_{n}: B \rightarrow F\left(\phi_{n}(B), B\right)$ and $M_{n}: B \rightarrow F\left(\phi_{n}(1), B\right)$ agree. A simple calculation of the Hessian of $M_{n}$ gives the result:

$$
\begin{aligned}
M_{n}\left(e^{i \alpha}\right) & =-\frac{1}{2} \sum_{[x, y] \in \vec{E}} h_{x, y}\left|\phi_{n}(x)-e^{i \alpha_{x, y}} \phi_{n}(y)\right|^{2}+\sum_{x \in X} V_{x}\left|\phi_{n}(x)\right|^{2} \\
& =-\sum_{[x, y] \in E} h_{x, y}\left(\phi_{n}(x)^{2}+\phi_{n}(y)^{2}-2 \cos \alpha_{x, y} \phi_{n}(x) \phi_{n}(y)\right)+\sum_{x \in X} V_{x}\left|\phi_{n}(x)\right|^{2} .
\end{aligned}
$$

Computing the second derivative with respect to $\alpha$ at $\alpha=0$ gives $\operatorname{Hessian}\left(M_{n}\right)=Q(\alpha)$.

\section{Appendix A: A pedestrian approach to the calculus of the Hessian of $\Lambda_{n}$ in Section 5}

We will derive a direct approach to the calculus of the second derivative of an eigenvalue which could be used directly in the proof of Lemma 7. Let $t \rightarrow A(t)$ be a $C^{2}$ curve defined near $t=0$ in the space of Hermitian matrices on a finite-dimensional Hilbert space $(\mathscr{H},\langle\cdot \mid \cdot\rangle)$. Let us assume that $\lambda(0)$ is an eigenvalue of $A(0)$ of multiplicity one with a normalized eigenvector $\phi(0)$. Then, for $t$ close to $0, A(t)$ has a simple eigenvalue $\lambda(t)$ of multiplicity one which is a $C^{2}$ function of $t$. We can choose an associated eigenfunction $\phi(t)$ which is $C^{2}$ with respect to $t$. The following assertions give the values of the first and second derivatives of $\lambda(t)$ at $t=0$ : 
Proposition 1. Under the previous assumptions, we have

$$
\lambda^{\prime}(0)=\left\langle A^{\prime}(0) \phi(0) \mid \phi(0)\right\rangle .
$$

If $\lambda^{\prime}(0)=0$, we have

$$
\lambda^{\prime \prime}(0)=\left\langle A^{\prime \prime}(0) \phi(0) \mid \phi(0)\right\rangle+2\left\langle\phi^{\prime}(0) \mid A^{\prime}(0) \phi(0)\right\rangle,
$$

where $\phi^{\prime}(0)$ is any solution of $(A(0)-\lambda(0)) \phi^{\prime}(0)=-A^{\prime}(0) \phi(0)$.

In particular, if $A^{\prime}(0) \phi(0)=0$,

$$
\lambda^{\prime \prime}(0)=\left\langle A^{\prime \prime}(0) \phi(0) \mid \phi(0)\right\rangle .
$$

Proof. We start with $(A(t)-\lambda(t)) \phi(t)=0$ where $\phi(t)$ is an eigenfunction of $A(t)$ which depends in a $C^{2}$ way on $t$. Taking the first derivative, we get

$$
\left(A^{\prime}(t)-\lambda^{\prime}(t)\right) \phi(t)+(A(t)-\lambda(t)) \phi^{\prime}(t)=0 .
$$

Putting $t=0$ and taking the scalar product with $\phi(0)$, we get the formula for $\lambda^{\prime}(0)$. Similarly, the $t$-derivative of (3) is

$$
\left(A^{\prime \prime}(t)-\lambda^{\prime \prime}(t)\right) \phi(t)+2\left(A^{\prime}(t)-\lambda^{\prime}(t)\right) \phi^{\prime}(t)+(A(t)-\lambda(t)) \phi^{\prime \prime}(t)=0 .
$$

Putting $t=0$, taking the scalar product with $\phi(0)$ and using $\lambda^{\prime}(0)=0$, we get the result.

We can apply this to $A(t):=H_{e^{i t \alpha}}$ with $\alpha \in \operatorname{ker} d^{\star}$ in order to get the Hessian of $\Lambda_{n}$ in Section 5. The condition $A^{\prime}(0) \phi(0)=0$ is exactly $d^{\star} \alpha=0$ !

\section{Appendix B: The case where the eigenfunction vanishes at some vertex}

In this appendix, we take $H \in O_{G}$ and assume that $\lambda_{n}=0$ is nondegenerate eigenvalue of $H$ with a normalized eigenfunction $\phi$. We have:

Proposition 2. Let us assume that, for all vertices $x$ satisfying $\phi(x)=0$, there exists a vertex $y \sim x$ so that $\phi(y) \neq 0$. Then, for any $\psi \in \mathbb{R}^{X}$ orthogonal to $\phi$, there exists a smooth deformation $H_{t} \in \mathrm{O}_{G}$ of $H$ so that $\dot{\phi}=\psi$.

It is enough to check that the space of $\dot{H} \phi$ is $\mathbb{R}^{X}$ and to use the first variation formulae given in Appendix A.

Theorem 3. Let us assume that the function $\phi$ vanishes at the unique vertex $x_{0}$. Then, the nullity of the Hessian of the "magnetic variation" of $H$ is at least $\left|n_{+}-n_{-}\right|$where $n_{ \pm}$is the number of vertices $x \sim x_{0}$ so that $\pm \phi(x)>0$.

Proof. Choose a smooth variation $H_{t}$ of $H$ so that $\dot{\phi}\left(x_{0}\right)=1$. Let $v$ be the number of sign changes of $\phi$ away from $x_{0}$. Then, for $t>0$ small enough, the number of sign changes of $\phi_{t}$ is $v+n_{-}$while, for $t<0$ small enough, it is $v+n_{+}$. We see from Theorem 1 that the magnetic Morse index is $v+n_{-}-(n-1)$ for $t>0$ and $v+n_{+}-(n-1)$. The discontinuity of the Morse index at $t=0$ is $\left|n_{+}-n_{-}\right|$. This gives the lower bound on the nullity.

Corollary 2. If $\left|n_{+}-n_{-}\right|>\beta$, the eigenvalue 0 is degenerate. 
Let us remark that this lower bound is not always sharp. In the following example, we have $n_{+}=n_{-}$, $\beta=2$ and the nullity of the Hessian is 2 .

Example B.1. The graph $G$ is made of 2 cycles of length 3 with a common vertex. The matrix of $H$ is chosen as follows:

$$
[H]=-\left(\begin{array}{lllll}
1 & 1 & 1 & 0 & 0 \\
1 & 1 & 2 & 0 & 0 \\
1 & 2 & 1 & 1 & 2 \\
0 & 0 & 1 & 1 & 1 \\
0 & 0 & 2 & 1 & 1
\end{array}\right) .
$$

Using the fact that the graph has a symmetry of order 2 exchanging the 2 cycles, one can split $\mathbb{R}^{X}$ and the matrix $H$ into the even and odd parts. This allows us to check that $\lambda_{4}=0$ is nondegenerate. In order to compute the magnetic Hessian, we check that it is possible to build a decomposition $\Omega^{1}(G)=d \mathbb{R}^{X} \oplus K$ which is $Q$-orthogonal and with $K \subset \operatorname{ker} d^{\star}$. It is then easy to check that the magnetic Hessian evaluated on $K$ vanishes.

\section{Acknowledgements}

Thanks to Roland Bacher, Gregory Berkolaiko and Françoise Truc for carefully checking the manuscript.

\section{References}

[Berkolaiko 2008] G. Berkolaiko, "A lower bound for nodal count on discrete and metric graphs", Comm. Math. Phys. 278:3 (2008), 803-819. MR 2008j:39019 Zbl 1171.05356

[Berkolaiko 2013] G. Berkolaiko, "Nodal count of graph eigenfunctions via magnetic perturbation", Anal. PDE 6:5 (2013), 1213-1233.

[Berkolaiko and Weyand 2012] G. Berkolaiko and T. Weyand, "Stability of eigenvalues of quantum graphs with respect to magnetic perturbation and the nodal count of the eigenfunctions", preprint, 2012. arXiv 1212.4475

[Burman 2012] Y. M. Burman, "Operators of rank 1, discrete path integration and graph Laplacians”, preprint, 2012. arXiv 1205.1123

[Colin de Verdière 1998] Y. Colin de Verdière, Spectres de graphes, Cours Spécialisés 4, Société Mathématique de France, Paris, 1998. MR 99k:05108 Zbl 0913.05071

[Colin de Verdière 2012] Y. Colin de Verdière, "Magnetic interpretation of the nodal defect on graphs", version 1, preprint, 2012. arXiv 1201.1110v1

[Courant and Hilbert 1953] R. Courant and D. Hilbert, Methods of mathematical physics, I, Interscience Publishers, New York, 1953. MR 16,426a Zbl 0051.28802

[Fiedler 1975] M. Fiedler, "Eigenvectors of acyclic matrices”, Czechoslovak Math. J. 25:4 (1975), 607-618. MR 52 \#8151 Zbl 0325.15014

[Forman 1993] R. Forman, "Determinants of Laplacians on graphs", Topology 32:1 (1993), 35-46. MR 94g:58247 Zbl 0780. 05041

[Kenyon 2012] R. Kenyon, “The Laplacian on planar graphs and graphs on surfaces”, preprint, 2012. arXiv 1203.1256

Received 13 Dec 2012. Revised 15 Jan 2013. Accepted 14 Feb 2013.

YVES COLIN DE VERDIÈRE: yves.colin-de-verdiere@ujf-grenoble.fr

Unité Mixte de Recherche CNRS-UJF 5582, Institut Fourier, BP 74, 38402 Saint Martin d'Hères cedex, France

http://www-fourier.ujf-grenoble.fr/ ycolver/ 


\title{
Analysis \& PDE
}

\author{
msp.org/apde
}

\section{EDITORS}

EDITOR-IN-CHIEF

\author{
Maciej Zworski \\ zworski@math.berkeley.edu \\ University of California \\ Berkeley, USA
}

BOARD OF EDITORS

Nicolas Burq Université Paris-Sud 11, France

nicolas.burq@math.u-psud.fr

Sun-Yung Alice Chang Princeton University, USA

chang@math.princeton.edu

Michael Christ University of California, Berkeley, USA

mchrist@math.berkeley.edu

Charles Fefferman Princeton University, USA

cf@math.princeton.edu

Ursula Hamenstaedt Universität Bonn, Germany

ursula@math.uni-bonn.de

Vaughan Jones U.C. Berkeley \& Vanderbilt University vaughan.f.jones@vanderbilt.edu

Herbert Koch Universität Bonn, Germany koch@math.uni-bonn.de

Izabella Laba University of British Columbia, Canada ilaba@math.ubc.ca

Gilles Lebeau Université de Nice Sophia Antipolis, France lebeau@unice.fr

László Lempert Purdue University, USA lempert@math.purdue.edu

Richard B. Melrose Massachussets Institute of Technology, USA rbm@math.mit.edu

Frank Merle Université de Cergy-Pontoise, France Frank.Merle@u-cergy.fr

William Minicozzi II Johns Hopkins University, USA minicozz@math.jhu.edu

Werner Müller Universität Bonn, Germany mueller@math.uni-bonn.de
Yuval Peres

Gilles Pisier

Tristan Rivière

Igor Rodnianski

Wilhelm Schlag

Sylvia Serfaty

Yum-Tong Siu

Terence Tao

Michael E. Taylor

Gunther Uhlmann

András Vasy

Dan Virgil Voiculescu

Steven Zelditch
University of California, Berkeley, USA

peres@stat.berkeley.edu

Texas A\&M University, and Paris 6

pisier@math.tamu.edu

ETH, Switzerland

riviere@math.ethz.ch

Princeton University, USA

irod@math.princeton.edu

University of Chicago, USA

schlag@math.uchicago.edu

New York University, USA

serfaty@cims.nyu.edu

Harvard University, USA

siu@math.harvard.edu

University of California, Los Angeles, USA

tao@math.ucla.edu

Univ. of North Carolina, Chapel Hill, USA

met@math.unc.edu

University of Washington, USA

gunther@math.washington.edu

Stanford University, USA

andras@math.stanford.edu

University of California, Berkeley, USA

dvv@math.berkeley.edu

Northwestern University, USA

zelditch@math.northwestern.edu

\section{PRODUCTION}

production@msp.org

Silvio Levy, Scientific Editor

See inside back cover or msp.org/apde for submission instructions.

The subscription price for 2013 is US \$160/year for the electronic version, and \$310/year ( $\$ 35$, if shipping outside the US) for print and electronic. Subscriptions, requests for back issues from the last three years and changes of subscribers address should be sent to MSP.

Analysis \& PDE (ISSN 1948-206X electronic, 2157-5045 printed) at Mathematical Sciences Publishers, 798 Evans Hall \#3840, c/o University of California, Berkeley, CA 94720-3840, is published continuously online. Periodical rate postage paid at Berkeley, CA 94704, and additional mailing offices.

APDE peer review and production are managed by EditFLOW ${ }^{\circledR}$ from Mathematical Sciences Publishers.

PUBLISHED BY

- mathematical sciences publishers

nonprofit scientific publishing

http://msp.org/

(C) 2013 Mathematical Sciences Publishers 


\section{ANALYSIS \& PDE}

\section{Volume $6 \quad$ No. $5 \quad 2013$}

A Lichnerowicz estimate for the first eigenvalue of convex domains in Kähler manifolds

1001

VINCENT GUEDJ, BORIS KOLEV and NADER YEGANEFAR

Sharp modulus of continuity for parabolic equations on manifolds and lower bounds for the 1013 first eigenvalue

Ben ANDREws and Julie ClutTERbuCK

Some minimization problems in the class of convex functions with prescribed determinant

NAM Q. LE and OVIDIU SAVIN

On the spectrum of deformations of compact double-sided flat hypersurfaces

1051

DENIS BORISOV and PEDRO FREITAS

Stabilization for the semilinear wave equation with geometric control condition

1089

ROMAIN JOLY and CAMILLE LAURENT

Instability theory of the Navier-Stokes-Poisson equations

JUHI JANG and IAN TICE

Dynamical ionization bounds for atoms

1183

ENNO LENZMANN and MATHIEU LEWIN

Nodal count of graph eigenfunctions via magnetic perturbation

GREGORY BERKOLAIKO

Magnetic interpretation of the nodal defect on graphs

YVES COLIN DE VERDIÈRE 